\title{
Simulation Studies Of Photon Signal Reconstruction In The DUNE Single Phase Far Detector With Xe Doping
}

\section{Whitmaur Castiglioni1,2 - SULI Program, Alex Himmel², Bryan Ramson²}

${ }^{1}$ Illinois Institute of Technology, ${ }^{2}$ Fermi National Accelerator Laboratory, *corresponding author: wcastiglioni@hawk.it.edu

\section{Abstract}

Argon when doped with Xenon has the potential to increase the effectiveness of scintillation systems in liquid argon time projection chambers (LArTPC). To test the potential improvements, a simulation study was conducted. A simulation of light going through liquid Argon and Xenon photon libraries (photon libraries are used to reduce the amount of computational power needed for interactions that produce a large amount of photons), comparing differences in their properties. The study found more light being emitted in a shorter time by $X e$ than by Ar with less attenuation present. Preliminary results show lower efficiency of $\mathrm{Ar}$ at closer distances. It is unclear whether this is due to inherit properties of the $\mathrm{Xe}$ or a result of selection cuts not being optimized. These potential improvements are being looked into for future neutrino experiments such as the Deep Underground Neutrino Experiment (DUNE).

\section{Introduction}

Xenon possess the ability when added in small amounts to Argon to improve the performance of a liquid argon based detector. Previous experimental studies have shown improvements in energy resolution, spatial and time resolution, and pulse shape discrimination (a metric that is important in dark matter studies). These improvements are due to the way $X e$ changes decay times and scattering. When either Ar or Xe are excited an excimer can form (an excimer being a metastable atom). These excimers bond with ground state atoms and produce light when they decay. Ions can recombine with electrons to form excimers. These excimers for both $\mathrm{Xe}$ and $\mathrm{Ar}$ exist in two states known as singlet or triplet states. For Xe both of these states decay faster then the corresponding Ar states. When producing scintillation light, Ar emits a wavelength of $128_{\mathrm{nm}}$ while Xe scintillation light is $175_{\mathrm{nm}}$. Scattering in LAr is dependent on Rayleigh scattering, with scattering $\propto 1 / \lambda^{4}$ leading with reduced scattering for Xe.

\section{Analysis and Results}

A simulation of $20 \mathrm{MeV}$ energy electrons and radiologicals simulating results from supernova neutrinos going through a pure $\mathrm{Xe}$ and Ar photon library produced the data for our analysis. Analyzing how the flashes (flashes being a way of grouping signals from light) were split up between $\mathrm{Ar}$ and $X e$ for both the OP flash and OP slice algorithms showed $X e$ in both cases not being split up to the same extent as Ar. This is most likely due to the faster decay times for both the fast and slow decay times for $\mathrm{Xe}$. The difference between these two algorithms can be generalized to OP flash focusing only on the early light and OP slice capturing early and late light together from the optical signals. The simulated supernova data show less attenuation in efficiency for distance (likely due to $\mathrm{Xe}$ emitting a lower wavelength than $\mathrm{Ar}$ with the medium scattering the light according to Rayleigh scattering). Xe was slightly less efficient than Ar closer to the photon collection system. This suspected discrepancy for short distance in $\mathrm{Xe}$ is thought to be inappropriate selection cuts that should be improved with preliminary work being done to optimize these cuts.

\section{Conclusion}

These simulations have demonstrated that the flashes from $X e$ are less likely to be split up in either the OPFlash or OPSlice algorithm. It was also demonstrated that there is less efficiency attenuation with distance. However there needs to be more work done to conclusively determine the benefit of doping argon with xenon.

\section{Doping Liquid}

Argon With Xenon Has The Potential

To Improve

Photon Detector

Performance

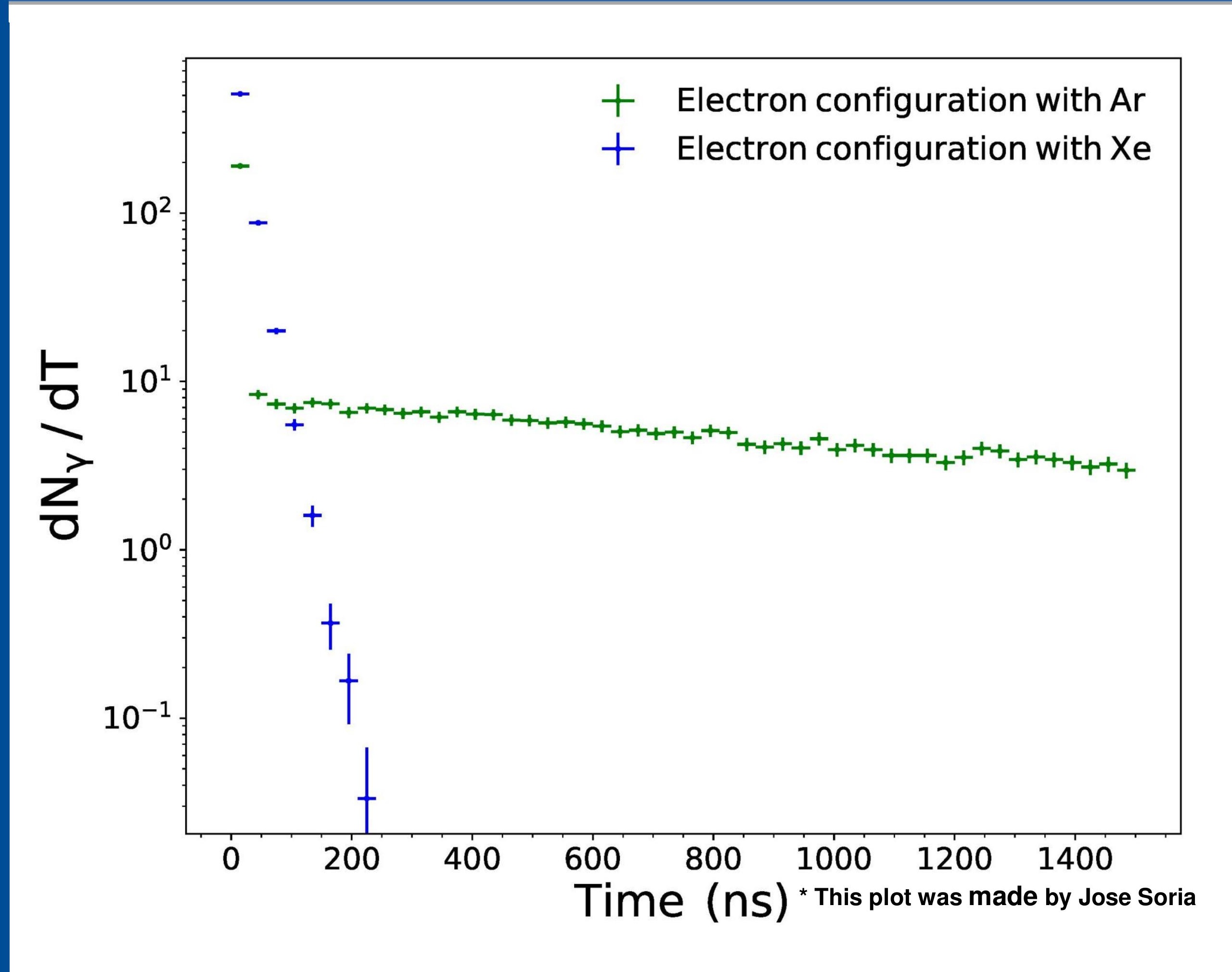

OP Flash and OP Slice are two different algorithms to split up the optical signal from the light. OP Flash only deals with the early light and OP Slice captures the early and late decay light light and
together.

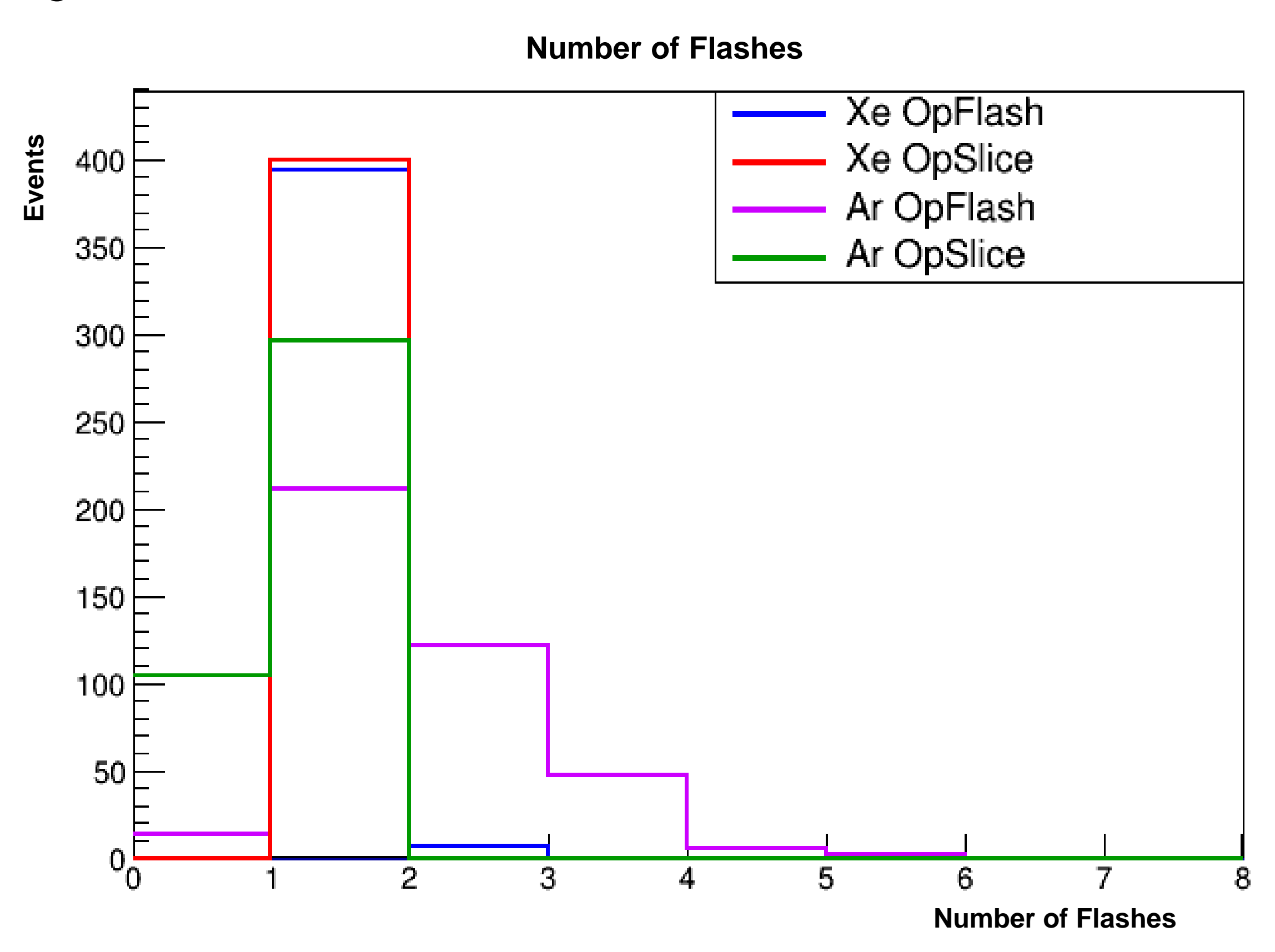

With both algorithms the light from $\mathrm{Xe}$ is not separated to the same extent as Ar.
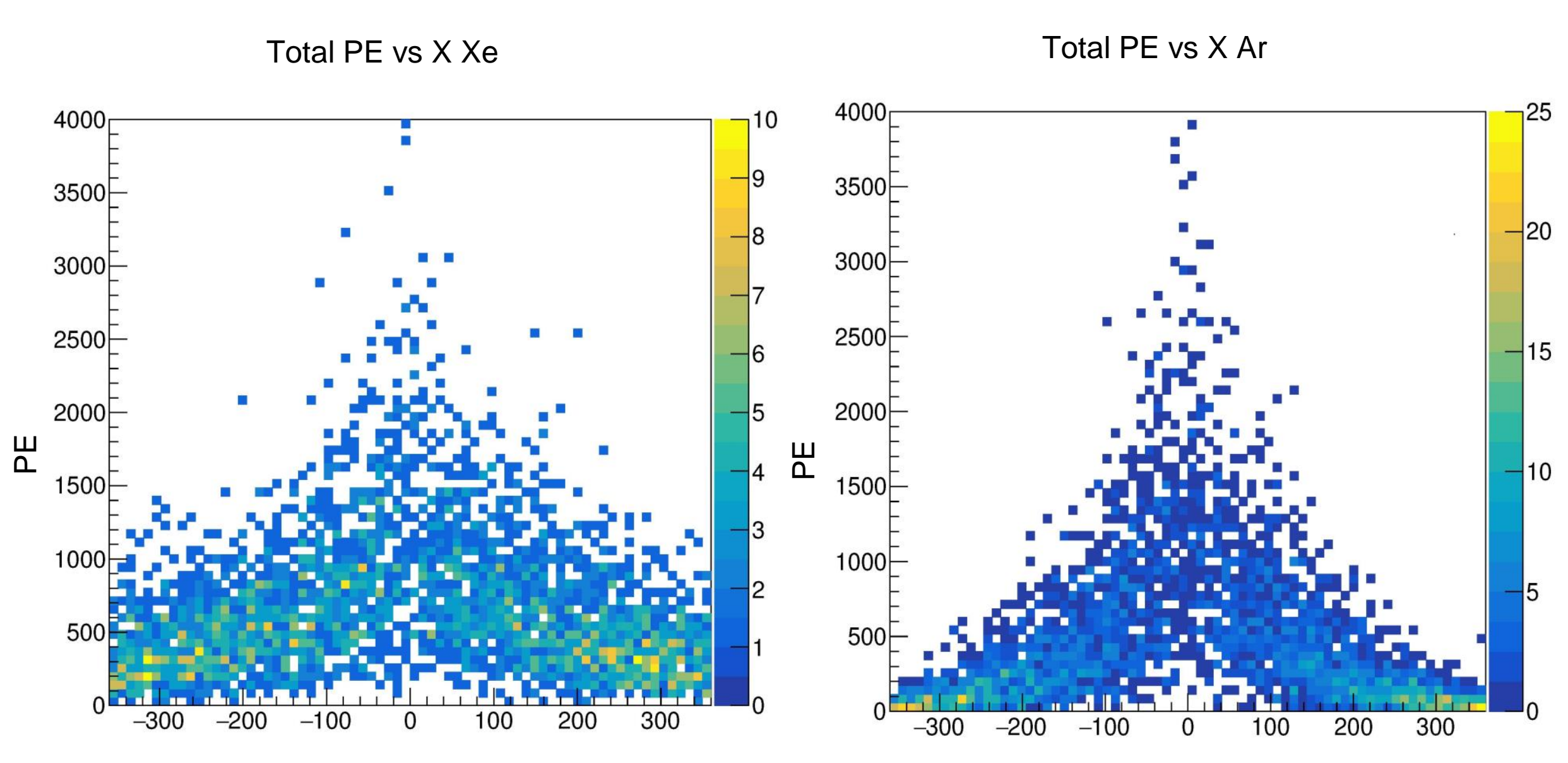

There is more light collected by $\mathrm{Xe}$ than $\mathrm{Ar}$ in the photon detection system from further away. $X$ in this case being the distance away from a wall of PMTs.

Xe is red Ar is blue

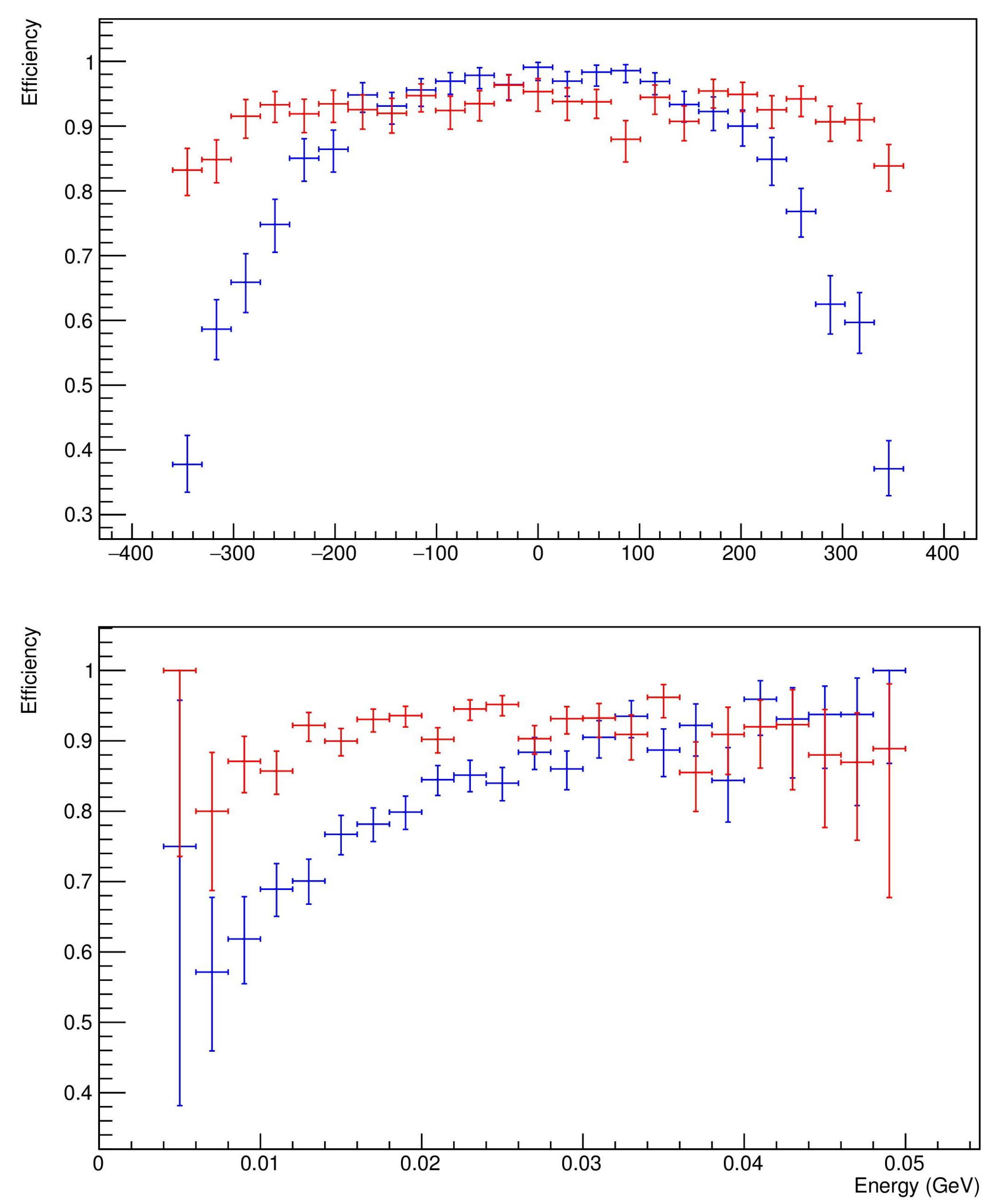

The efficiency plots for Xe currently use the largest flash as our selection parameter for $\mathrm{Xe}$ as the selection cuts still need to be optimized. 